\title{
WEIGHT STRUCTURE THEOREMS AND FACTORIZATION OF POSITIVE OPERATORS
}

\author{
STEVEN BLOOM AND RON KERMAN
}

(Communicated by Palle E. T. Jorgensen)

\begin{abstract}
We characterize the conditions under which weighted norm inequalities for a positive operator $T$ can be obtained by interpolation with change of measure. The results are applied to the construction of all good weight pairs for $T$. This construction is used to show that the study of weighted norm inequalities for operators $T$ that factor as $T=P Q$ reduce to that of the weighted norm inequalities for the factors $P$ and $Q$.
\end{abstract}

\section{INTRODUCTION}

Let $X$ and $Y$ be $\sigma$-finite measure spaces. Denote by $P(X)$ and $P(Y)$ the class of nonnegative (possibly infinite) measurable functions on $X$ and $Y$ respectively. Call an operator $T: P(X) \rightarrow P(Y)$ satisfying $T\left(f_{1}+c f_{2}\right)=$ $T f_{1}+c T f_{2}$ for each $f_{1}$ and $f_{2} \in P(X)$ and $c>0$, a positive linear operator. Suppose $T$ is such an operator from $P(X)$ to $P(Y)$ for which there is another operator, $T^{*}$, from $P(Y)$ to $P(X)$ conjugate to $T$; that is,

$$
\int_{X} f T^{*} g=\int_{Y} g T f \text { for each } f \in P(X) \text { and } g \in P(Y) \text {. }
$$

For example, given a measure $\nu$ on $X \times Y$, define

$$
T f(y)=\int_{X} f(x) d \nu(x, y), \quad T^{*} g(x)=\int_{Y} g(y) d \nu(x, y),
$$

where $f \in P(X)$ and $g \in P(Y)$.

We are interested in weighted norm inequalities of the form

$$
\left(\int_{Y}[v T f]^{q}\right)^{1 / q} \leq C\left(\int_{X}[u f]^{p}\right)^{1 / p} \text { for each } f \in P(X),
$$

where the weights $u$ and $v$ belong, respectively, to $P(X)$ and $P(Y)$, and the indices $p$ and $q \in[1, \infty]^{1}$. We refer to a pair of weights $(u, v)$ for which (1.3) holds as a good weight pair.

Received by the editors June 1, 1990.

1980 Mathematics Subject Classification (1985 Revision). Primary 47B38; Secondary 47A30.

The second author's research was supported in part by NSERC grant A4021.

${ }^{1}$ Here, and below, when, say, $p=\infty$, we replace the expression on the right side of (1.3) by ess $_{x \in X} \sup u(x) f(x)$. 
When one of $p$ and $q$ is either 1 or $\infty$, there are well known and simple characterizations of the good weight pairs. Aronszjan's generalization of Schur's Lemma and Gagliardo's converse [2], show that every inequality of the form (1.3) can be obtained as a consequence of these special ones, provided $p \geq q$. We reformulate this result for our setting in $\S 2$, and describe the extent to which such results apply when $p<q$.

In $\S 3$, a description of good weight pairs is given in such a way that the weights are determined by specifying certain "parameters"; this was the approach used by Helson and Szegö [3] to look at the weights for the conjugate function operator. We also use the description to show that the study of weighted norm inequalities for composite operators can be reduced to that of similar ones for the factors.

Given a weight $w \in P(X)$ and fixed $p \in[1, \infty]$, the weighted Lebesgue space $L^{p}(X, w)$ consists of certain measurable functions on $X$; specifically, $L^{p}(X, w)=\left\{f:\|w f\|_{p}<\infty\right\}$, where, as usual, $\|f\|_{p}=\left[\int_{X}|f(x)|^{p} d x\right]^{1 / p}$. The inequality (1.3) is equivalent to $T$ being in the space $\left[L^{p}(X, u), L^{q}(Y, v)\right]$ of bounded linear operators from $L^{p}(X, u)$ to $L^{q}(Y, v)$, and it is often convenient to think of (1.3) in this way.

\section{DiRECT AND INVERSE INTERPOLATION}

We begin by reformulating (and generalizing slightly) Theorems 1. I and 1.II of [2] to show, in effect, that (1.3) can always be obtained by interpolation with change of measure when $p \geq q$.

Theorem 2.1. Let $X$ and $Y$ be $\sigma$-finite measure spaces, and let $u \in P(X)$, $v \in P(Y)$ be weight functions. Suppose $T$ is a positive linear operator having conjugate $T^{*}$ and satisfying the property that $\left\{f_{n}\right\} \in P(X), f_{n} \uparrow f$ implies $T f_{n} \uparrow T f$. Fix $p$ and $q$ with $1<q \leq p<\infty$. Then the following are equivalent.

(a) $T \in\left[L^{p}(X, u), L^{q}(Y, v)\right]$.

(b) There exist positive functions $\alpha \in L^{p q^{\prime}}(X)$ and $\beta \in L^{p q^{\prime}}(Y)$ and a constant $C>0$, such that

(i) $\quad T\left(\alpha^{q^{\prime}} u^{-1}\right) \leq C \beta^{p\left(q^{\prime}-1\right)} v^{-1}$

(ii) $T^{*}\left(\beta^{p} v\right) \leq C \alpha^{(p-1) q^{\prime}} u$.

(Here the prime denotes the conjugate exponent, $1 / r+1 / r^{\prime}=1$.)

(c) There exist positive functions $\alpha \in P(X)$ and $\beta \in P(Y)$ such that

(ii) $\int_{Y}\left[\beta^{-q^{\prime}} v T\left(\alpha^{q^{\prime}} u^{-1}\right)\right]^{r}<\infty$,

where $1 / r=1 / q-1 / p$. 
When $X=Y$ we may take $\alpha=\beta$ in (b) and (c).

This is a fairly straightforward generalization of the Azonszjan and Gagliardo Theorems to the weighted setting, and we omit its proof. Similar results are proven in [1] and [5] when $p=q$, and in [4] when $p>q$.

The restrictions $\alpha \in L^{p q^{\prime}}(X)$ and $\beta \in L^{p q^{\prime}}(Y)$ are necessary in (b) of the theorem if $p>q$. Thus, while the identity operator satisfies (2.2) with $u=$ $v=\alpha=\beta=1$ when $X=Y$, the inclusion $L^{p}(X) \subset L^{q}(X)$ holds if and only if $X$ is a finite measure space.

Now we study the extent to which there is an analogue of Theorem 2.1 when $p<q$. This is done for positive integral operators; in the notation of (1.2)

$$
d \nu(x, y)=K(x, y) d x d y,
$$

when the kernel $K \in P(X \times Y)$. For $r \in(1, \infty)$, denote by $T_{r}$ the positive integral operator from $P(X)$ to $P(Y)$ with kernel $K_{r}(x, y)=K(x, y)^{r}$.

The following lemma is due to M. Cwikel.

Lemma 2.3. Let $X, Y, u$, and $v$ be as in Theorem 2.1. Suppose $T$ is a positive integral operator from $P(X)$ to $P(Y)$ with kernel $K$. Fix $r \in(1, \infty)$. Then,

(a) $T \in\left[L^{1}(X, u), L^{r}(Y, v)\right]$ if and only if

$$
u^{-r} T_{r}^{*} v^{r} \leq C<\infty
$$

that is,

$$
T_{r} \in\left[L^{1}\left(X, u^{r}\right), L^{1}\left(Y, v^{r}\right)\right] .
$$

(b) $T \in\left[L^{r}(X, u), L^{\infty}(Y, v)\right]$ if and only if

$$
v^{r^{\prime}} T_{r^{\prime}} u^{r^{\prime}} \leq C<\infty
$$

that is,

$$
T_{r^{\prime}} \in\left[L^{\infty}\left(X, u^{r^{\prime}}\right), L^{\infty}\left(Y, v^{r^{\prime}}\right)\right] .
$$

Proof. We need only prove (b) because (a) follows by duality. Now $T \in$ $\left[L^{r}(X, u), L^{\infty}(Y, v)\right]$ if and only if

$$
v(y)(T f)(y) \leq C\left(\int_{x}[u f]^{r}\right)^{1 / r} \text { for each } f \in P(X), y \in Y .
$$

Letting $F=u f$, so that $F \in L^{r}(X),(2.6)$ becomes

$$
v(y) \int_{X} K(x, y) u(x)^{-1} F(x) d x \leq C\left(\int_{X} F^{r}\right)^{1 / r} \quad \text { for each } y \in Y .
$$

Taking the supremum over all $F \in L^{r}(X)$ with $\|F\|_{r}=1,(2.7)$ is seen to be equivalent to

$$
v(y)\left[\int_{X} K(x, y)^{r^{\prime}} u(x)^{-r^{\prime}} d x\right]^{1 / r^{\prime}} \leq C<\infty \quad \text { or } \quad v^{r^{\prime}} T_{r^{\prime}} u^{r^{\prime}} \leq C<\infty .
$$


Theorem 2.8. Let $X, Y, u, v, T$, and $T_{r}$ be as in the Lemma 2.3. Fix $p$ and $q$ with $1<p<q<\infty$ and define $r$ by $1 / r=1 / p-1 / q$. Suppose

$$
T \in\left[L^{p}(X, u), L^{q}(Y, v)\right]
$$

can be proved using interpolation with change of measure; that is,

$$
u=u_{0}^{1-t} u_{1}^{t} \text { and } v=v_{0}^{1-t} v_{1}^{t} \text { where } t=r^{\prime} / p^{\prime},
$$

for some $u_{i} \in P(X)$ and $v_{i} \in P(Y), i=0,1$, where

$$
\begin{aligned}
& \text { (i) } T \in\left[L^{1}\left(X, u_{0}\right), L^{r^{\prime}}\left(Y, v_{0}\right)\right] \\
& \text { (ii) } T \in\left[L^{r}\left(X, u_{1}\right), L^{\infty}\left(Y, v_{1}\right)\right] .
\end{aligned}
$$

Then,

$$
T_{r^{\prime}} \in\left[L^{q / r^{\prime}}\left(X, u^{r^{\prime}}\right), L^{q / r^{\prime}}\left(Y, v^{r^{\prime}}\right)\right] .
$$

Again, (2.12) implies (2.9).

Proof. Assume (2.10) and (2.11) hold. By Lemma 2.3, (2.11) implies

$$
T_{r^{\prime}} \in\left[L^{1}\left(X, u_{0}^{r^{\prime}}\right), L^{1}\left(Y, v_{0}^{r^{\prime}}\right)\right] \text { and } T_{r^{\prime}} \in\left[L^{\infty}\left(X, u_{1}^{r^{\prime}}\right), L^{\infty}\left(Y, v_{1}^{r^{\prime}}\right)\right] .
$$

Interpolating with change the measure [6], and taking $t=r^{\prime} / p^{\prime}$, we obtain (2.12).

Next, suppose we have (2.12). Let $f \in P(X)$ with $\|u f\|_{p}=1$. Then,

$$
\begin{aligned}
T f & =\int_{X}\left[K u^{-p / r} f^{1-p / r}\right][f u]^{p / r} \\
& \leq\left[\int_{X} K^{r^{\prime}}\left(u^{-p / r} f^{1-p / r}\right)^{r^{\prime}}\right]^{1 / r^{\prime}}, \quad \text { by Hölder's inequality, } \\
& =\left(T_{r^{\prime}} g\right)^{1 / r^{\prime}},
\end{aligned}
$$

where $g=\left[u^{-p / r} f^{1-p / r}\right]^{r^{\prime}}$. Notice that $\left(u^{r^{\prime}} g\right)^{q / r^{\prime}}=(u f)^{p}$; so $g \in L^{q / r^{\prime}}\left(X, u^{r^{\prime}}\right)$. Thus by (2.12),

$$
\begin{aligned}
\|v T f\|_{q} & \leq\left\|\left(v^{r^{\prime}} T_{r^{\prime}} g\right)^{1 / r^{\prime}}\right\|_{q} \\
& =\left\|v^{r^{\prime}} T_{r^{\prime}} g\right\|_{q / r^{\prime}}^{1 / r^{\prime}} \leq C\left\|u^{r^{\prime}} g\right\|_{q / r^{\prime}}^{1 / r^{\prime}}=C\|u f\|_{p}^{p / q}=C .
\end{aligned}
$$

Here we point out that for examples like the fractional integral, with kernel $K(x, y)=|x-y|^{\alpha-n}$ for fixed $\alpha \in(0, n)$, when $X=Y=\mathbb{R}^{n}$, and $u=v=1$, the Sobolev embedding theorem, which asserts that $T \in\left[L^{p}, L^{q}\right]$ when $1 / q=$ $1 / p-\alpha / n$, cannot be obtained by interpolation with change of measure, since neither (2.11) nor (2.12) can hold for nontrivial weights when $K(x, y)^{r^{\prime}}=$ $|x-y|^{-n}$.

\section{STRUCTURE OF GOOD WEIGHT PAIRS AND FACTORIZATION}

Our main result is the following description of all good weight pairs for positive linear operators. 
Theorem 3.1. Let $X$ and $Y$ be $\sigma$-finite measure spaces and $T$ a positive linear operator from $P(X)$ to $P(Y)$ with conjugate $T^{*}$. Fix $p$ and $q \in(1, \infty)$, $p \geq q$. Then

(a) Suppose $f, \lambda \in P(X)$ and $g, \mu \in P(Y)$, where

$$
\begin{aligned}
f, T^{*} g^{p}>0 \text { a.e. on } X, & g, T f^{q^{\prime}}>0 \text { a.e. on } Y, \\
\lambda \in L^{\infty}(X), & \mu \in L^{\infty}(Y),
\end{aligned}
$$

and

$$
\int_{X} f^{q^{\prime}} T^{*} g^{p}=\int_{Y} g^{p} T f^{q^{\prime}}<\infty .
$$

Define the weights $u \in P(X)$ and $v \in P(Y)$ by

$$
u^{-p}=\lambda f^{(p-1) q^{\prime}} / T^{*} g^{p} \text { and } v^{q^{\prime}}=\mu g^{p\left(q^{\prime}-1\right)} / T f^{q^{\prime}} .
$$

Then, $T \in\left[L^{p}(X, u), L^{q}(Y, v)\right]$.

(b) Conversely, suppose $T \in\left[L^{p}(X, u), L^{q}(Y, v)\right]$ for fixed weights $u \in$ $P(X)$ and $v \in P(Y)$. Let $E=\operatorname{supp} v T\left(u^{-1}\right)$ and $F=\operatorname{supp} u^{-1} T^{*}(v)$. Then, there exist $f, \lambda \in P(X)$ and $g, \mu \in P(Y)$ with

$$
\begin{aligned}
f, T^{*} g^{p}>0 \text { a.e. on } E, & g, T f^{q^{\prime}}>0 \text { a.e. on } F \\
\lambda \in L^{\infty}(X), & \mu \in L^{\infty}(Y)
\end{aligned}
$$

such that (3.2) holds and such that the weights $u$ and $v$ are given by (3.3) on $E$ and $F$ respectively.

Proof. (a) is an easy consequence of the special case $\lambda \equiv 1$ on $X$ and $\mu \equiv 1$ on $Y$, which we now consider. Define $\alpha$ and $\beta$ by

$$
\alpha^{p q^{\prime}}=f^{q^{\prime}} T^{*} g^{p}=u^{p} f^{p q^{\prime}}
$$

and

$$
\beta^{p q^{\prime}}=g^{p} T f^{q^{\prime}}=v^{-q^{\prime}} g^{p q^{\prime}} .
$$

Then, $\alpha \in L^{p q^{\prime}}(X)$ and $\beta \in L^{p q^{\prime}}(Y)$ by (3.2). It follows that (2.2) holds with $C=1$, since

$$
\begin{aligned}
T\left(\alpha^{q^{\prime}} u^{-1}\right) & =T f^{q^{\prime}} \quad \text { by }(3.5) \\
& =\beta^{p q^{\prime}} g^{-p}=\beta^{p\left(q^{\prime}-1\right)} v^{-1} \text { by (3.6). }
\end{aligned}
$$

Similarly,

$$
T^{*}\left(\beta^{p} v\right)=\alpha^{(p-1) q^{\prime}} u
$$

To prove (b), let $\alpha \in L^{p q^{\prime}}(X)$ and $\beta \in L^{p q^{\prime}}(Y)$ be the positive functions guaranteed by Theorem 2.1 to satisfy (2.2). Then, (3.4) holds for the functions $f$ and $g$ defined by

$$
f^{q^{\prime}}=\alpha^{q^{\prime}} u^{-1} \cdot \chi_{E}
$$


and

$$
g^{p}=\beta^{p} v \cdot \chi_{F}
$$

Further, (3.2) holds, since, for example,

$$
\begin{aligned}
f^{q^{\prime}} T^{*} g^{p} & =f^{q^{\prime}} T^{*}\left(\beta^{p} v\right) \quad \text { by }(3.8) \\
& \leq C f^{q^{\prime}} \alpha^{(p-1) q^{\prime}} u \quad \text { by }(2.2)(\mathrm{ii}) \\
& \leq C \alpha^{p q^{\prime}} \text { by (3.7) }
\end{aligned}
$$

From this calculation, it also follows that if we define $\lambda$ by

$$
\lambda=\alpha^{-p q^{\prime}} f^{q^{\prime}} T^{*} g^{p},
$$

then $\lambda \in L^{\infty}(X)$. Similarly, setting

$$
\mu=\beta^{-p q^{\prime}} g^{p} T f^{q^{\prime}},
$$

we have $\mu \in L^{\infty}(Y)$. Finally,

$$
\begin{aligned}
u^{-p} & =\alpha^{-p q^{\prime}} f^{p q^{\prime}} \quad \text { by }(3.7) \\
& =\alpha^{-p q^{\prime}} f^{q^{\prime}} f^{(p-1) q^{\prime}} \\
& =\lambda f^{(p-1) q^{\prime}} / T^{*} g \text { on } E
\end{aligned}
$$

and, similarly,

$$
v^{q^{\prime}}=\mu g^{p\left(q^{\prime}-1\right)} / T f^{q^{\prime}} \text { on } F
$$

As an application of this result, we describe how to "factor" weighted norm inequalities.

Theorem 3.2. Let $X, Y$, and $Z$ be $\sigma$-finite measure spaces, and let $u \in P(X)$, $w \in P(Y)$ be weight functions. Suppose $S$ is a positive linear operator from $P(X)$ to $P(Y)$ having conjugate $S^{*}$ and satisfying $\left\{f_{n}\right\} \in P(X), f_{n} \uparrow f$ implies $S f_{n} \uparrow S f$; suppose $T$ is a similar operator from $P(Y)$ to $P(Z)$. Fix $p, q$, and $r$ with $1<r \leq q \leq p<\infty$. Then, a necessary and sufficient condition that the operator $T S \in\left[L^{p}(X, u), L^{r}(Z, w)\right]$ is the existence of $v \in P(Y)$ such that $S \in\left[L^{p}(X, u), L^{q}(Y, v)\right]$ and $T \in\left[L^{q}(Y, v), L^{r}(z, w)\right]$.

Proof. Only the necessity requires proof. For simplicity, we only consider the case in which $\operatorname{supp} S=X, \operatorname{supp} T=Y$, and $0<u, w<\infty$. By Theorem 3.1(b), there exist $f, \lambda \in P(X)$ and $g, \mu \in P(Z)$ with

$$
\begin{gathered}
f, S^{*} T^{*} g^{p}>0 \quad \text { a.e. on } X, \quad g, T S f^{r^{\prime}}>0 \text { a.e. on } Z \\
\lambda \in L^{\infty}(X), \quad \mu \in L^{\infty}(X)
\end{gathered}
$$

and

$$
\int_{X} f^{r^{\prime}} S^{*} T^{*} g^{p}=\int_{Z} g^{p} T S f^{r^{\prime}}<\infty,
$$


such that

$$
u^{-p}=\lambda f^{(p-1) r^{\prime}} / S^{*} T^{*} g^{p} \text { and } \quad w^{r^{\prime}}=\mu g^{p\left(r^{\prime}-1\right)} / T S f^{r^{\prime}} .
$$

Define $v$ by

$$
v^{q}=\left(T^{*} g^{p}\right)\left(S f^{r^{\prime}}\right)^{1-q}
$$

Set

$$
h=f^{r^{\prime} / q^{\prime}} \text { and } k=\left(T^{*} g^{p}\right)^{1 / p} \text {. }
$$

Then

$$
u^{-p}=\lambda h^{(p-1) q^{\prime}} / S^{*} k^{p} \quad \text { and } \quad v^{q^{\prime}}=\mu k^{p\left(q^{\prime}-1\right)} / S h^{q^{\prime}}
$$

moreover,

$$
\int_{X} h^{q^{\prime}} S^{*} k^{p}=\int_{X} f^{r^{\prime}} S^{*} T^{*} g^{p}<\infty .
$$

And so, $S \in\left[L^{p}(X, u), L^{q}(Y, v)\right]$, by Theorem 3.1(a).

This time taking

$$
h=\left(S f^{r^{\prime}}\right)^{1 / r^{\prime}} \text { and } k=g^{p / q},
$$

we have

$$
v^{-q}=h^{(q-1) r^{\prime}} / T^{*} k^{q} \text { and } \quad w^{r^{\prime}}=\mu k^{q\left(r^{\prime}-1\right)} / T h^{r}
$$

further,

$$
\int_{Z} k^{q} T h^{r^{\prime}}=\int_{Z} g^{p} T S f^{r^{\prime}}<\infty .
$$

Thus, $T \in\left[L^{q}(Y, v), L^{r}(Z, w)\right]$, and the proof is complete.

\section{REFERENCES}

1. S. Bloom, Solving weighted norm inequalities using the Rubio de Francia algorithm, Proc. Amer. Math. Soc. 101 (1987), 306-312.

2. E. Gagliardo, On integral transformations with positive kernel, Proc. Amer. Math. Soc. 16 (1965), 429-434.

3. H. Helson and G. A. Szegö, A problem in prediction theory, Ann. Math. Pura. Appl. (4) 51 (1960), 107-138.

4. E. Hernandez, Weighted inequalities through factorization, preprint.

5. B. Jawerth, Weighted inequalities for maximal operators: linearization, localization and factorization, Amer. J. Math. 108 (1986), 361-414.

6. E. M. Stein and G. Weiss, Interpolation of operators with change of measure, Trans. Amer. Math. Soc. 87 (1958), 159-172.

Department of Mathematics, Siena College, Loudonville, New York 12211

Department of Mathematics, Brock University, St. Catharines, Ontario, Canada 